\title{
ASSOCIATION STYDY OF SIRTUIN 1 POL YMORPHISMS WITH BONE MINERAL DENSITY AND BODY MASS INDEX
}

María T. Zarrabeitia, Carmen Valero, Juan C. Martín-Escudero, José M. Olmos, Alfonso Bolado-Carrancio, Eva L. de Sande-Nacarino, José C. Rodríguez-Rey, Jesús Sainz, and José A. Riancho

Unit of Legal Medicine, University of Cantabria. Santander, Spain. (M.T.Z.)

Department of Internal Medicine, Hospital U.M. Valdecilla-IFIMAV, University of Cantabria. RETICEF. Santander, Spain. (C.V., J.M.O., J.A.R.)

Service of Internal Medicine. Hospital U. Río Hortega, University of Valladolid. Valladolid, Spain. (J.C.M.E., E.L.S.N.)

Institute of Biomedicine and Biotechnology of Cantabria (IBBTEC), Spanish National Research Council (CSIC)-University of Cantabria. Santander, Spain. (J.S.)

Department of Biochemistry and Molecular Biology. University of Cantabria. Santander, Spain (A.B.C., J.C.R.R.)

Running title: sirtuin polymorphisms and BMI

Correspondence and reprint requests:

José A. Riancho

Dep. Internal Medicine

Hospital U.M. Valdecilla

Avda Valdecilla s/n

39008 Santander, Spain

Fax 34942201695

Tel 34942201990

Email: rianchoj@unican.es 


\section{ABSTRACT}

Background. Sirtuin 1, encoded by the SIRT1 gene, is an emerging modulator of carbohydrate and lipid metabolism and may also influence the differentiation of bone cells.

Aim. Our objective was to test the hypothesis that polymorphisms of SIRT1 are associated with body mass index (BMI) and bone mineral density (BMD).

Methods. Cross-sectional genetic association study, with genotyping of 10 single nucleotide polymorphisms of the SIRT1 region. The discovery cohort included 1394 individuals (342 men, 1052 women). Significant results were replicated in an independent cohort of 408 men.

Results. We did not find a significant association of genotypes with BMD. There were no significant BMI differences across genotypes in women either. However, in men, two polymorphisms tended to be associated with $\mathrm{BMI}$ in the discovery cohort ( $\mathrm{p}=0.03$ and 0.05$)$. A similar trend was also observed in the replication cohort. Thus, in the combined analysis of both cohorts men with C alleles at the rs 12049646 locus had a lower BMI than TT homozygotes, with a mean difference of $0.82 \mathrm{~kg} / \mathrm{m}^{2}$ (95\% confidence interval $0.15-1.48 ; \mathrm{p}=0.016$ ). Differences in the DNA binding of nuclear proteins between $\mathrm{C}$ and $\mathrm{T}$ alleles were also observed in vitro.

Conclusions. These results suggest that common variants of the SIRT1 gene influence BMI but not BMD.

KEYWORDS: sirtuins, osteoporosis, obesity, association study, gene expression, bone mineral density, body mass index. 


\section{INTRODUCTION}

Sirtuins deacetylate several histones and transcription factors, such as the ones encoded by

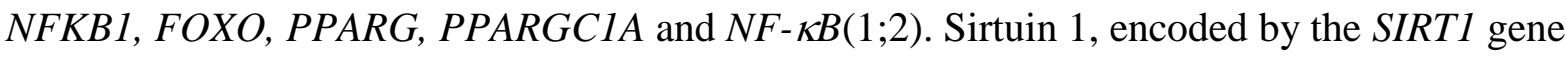
located on human chromosome 10q21.3, facilitates lipolysis in the adipose tissue, modulates the differentiation of adipocyte precursors, regulates fatty acid oxidation in the liver, and influences insulin secretion and resistance and the sensing of nutrient availability by the hypothalamus (37).

There is increasing evidence of interaction between bone, energy metabolism and adipose tissue $(8 ; 9)$. In fact, sirtuins may differentially drive the differentiation of mesenchymal precursors towards either the osteoblast or the adipocytic lineage, both in vitro and in vivo (10). Resveratrol, a natural inducer of the SIRT1 gene, promotes osteogenesis by inducing RUNX2 transcription (11) and inhibits osteoclast differentiation by interacting with the RANKL-NF- $\mathrm{KB}$ pathway $(12 ; 13)$.

Adiposity and bone mineral density (BMD) have a strong genetic component and hereditary factors explain about $50-80 \%$ of the interindividual variance $(14 ; 15)$. Genome-wide and candidate gene studies have identified several loci clearly associated with these phenotypes, but those loci only explain a small proportion of the interindividual variation in bone and body mass (15-17). Therefore, many more loci remain to be discovered. Since sirtuin 1 plays an important role as a metabolic regulator, we planned this study to check the hypothesis that common polymorphisms of SIRT1 are associated with body mass index (BMI) and BMD. 


\section{SUBJECTS AND METHODS}

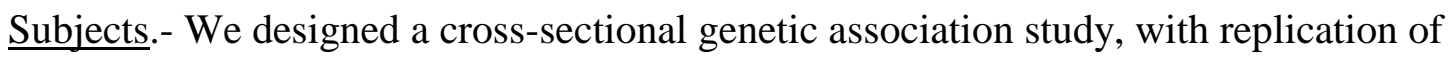
significant results in an independent group and in vitro studies of allelic differences in the binding of nuclear proteins.

The discovery cohort included 1394 Caucasians (342 men and 1052 women) from Cantabria, a region in Northern Spain, as previously described $(18 ; 19)$. All were interviewed by a physician to exclude drugs or diseases known to affect bone metabolism, such as hyperthyroidism, hyperparathyroidism, Cushing syndrome, immobilization, type I diabetes and other severe diseases (heart failure, chronic obstructive lung disease, malabsorption, liver disease, cancer, etc.). Body weight and height were measured by standard procedures and rounded to the nearest integer. Hip BMD was measured by DXA (Hologic).

The replication group included 408 males over 50 years of age living in Valladolid, a province in Central Spain. They were selected from the Hortega cohort, a general population sample extracted from the Valladolid area to study cardiovascular risk factors (20).

The study was approved by the Clinical Research Ethical Committees and informed consent was obtained from the participants.

Genotyping.- Single nucleotide polymorphisms (SNPs) were analyzed in blood DNA by using specific primers and Taqman probes (Taqman genotyping assays, Applied Biosystems, Foster City, CA). Tagger software (http://www.broadinstitute.org/mpg/tagger/) was used to select tag SNPs in the SIRT1 region plus 0.5kb downstream, and $30 \mathrm{~Kb}$ upstream (NCBI Build 35/UCSC hg17), with a minor allele frequency $>5 \%$ in the Caucasian population and $r^{2}>1$ as criteria. In addition, rs3740051 was also genotyped because bioinformatic analysis suggested that it included a putative transcription factor binding site. SNPs showing evidence for association in the discovery cohort were selected to be analyzed in the replication cohort. 
Gene expression and Electrophoretic Mobility Shift Assays (EMSA).- To study SIRT1

expression in bone, trabecular bone samples were obtained from the central part of the femoral heads of patients subjected to hip replacement because of osteoporotic hip fractures $(n=8)$ or controls with hip osteoarthritis $(\mathrm{n}=10)$. RNA was isolated with Trizol (Invitrogen, Carlsbad, CA, USA), and further purified by using a column adsorption procedure (Qiagen, Hilden, Germany). Aliquots of RNA (250 ng) were reverse-transcribed with the Superscript III kit (Invitrogen) and quantified by real-time PCR in an ABI7300 apparatus (Applied Biosystems), using specific primers and FAM-labelled probes for SIRT1 (Taqman gene expression assays, Applied Biosystems). The results were then normalized to the expression of the housekeeping gene TATA box binding protein $(T B P)$, as previously published $(21 ; 22)$.

Double-stranded oligonucleotides with the sequences including the region of the rs 12049646 polymorphism (TATATTGGTCTCGTTGGGATGTTC and

TATATTGGTCTTGTTGGGATGTTC) were used to determine the binding of $\mathrm{C}$ and $\mathrm{T}$ alleles respectively by EMSA. The forward oligonucleotides (the ones shown) were labelled in 5' with IRDye700 (Li-Cor Biosciences, Lincoln, NE) during the synthesis. The preparation of nuclear extracts and the electrophoresis procedures have been described previously (23). For the competition experiments, an excess of $\mathrm{C}$ allele oligonucleotide was added to the mix prior to the addition of the labelled oligonucleotide. The bands were quantified in an Odyssey Infrared Imaging System (Li-Cor Biosciences, Lincoln, NE). The inverse of band intensity versus the excess of unlabeled oligonucleotide was represented and the slope of the resulting straight line was inversely proportional to the affinity of each allele for the proteins in the nuclear extract. Statistical analysis.- The sample size was considered enough to have $>90 \%$ power to detect genetic effects explaining at least a $1 \%$ of the phenotype variance (estimated with Quanto software, available at http://hydra.usc.edu/gxe/). The departure from Hardy-Weinberg equilibrium (HWE) was tested with Plink software (24). The Haplotypic blocks were estimated 
by the Gabriel method, implemented in Haploview (25). The results were adjusted by potential confounding variables (age and weight) by ANCOVA or multiple linear regression. The results in different cohorts were combined by computing the mean difference with MIX software (26). The manuscript was elaborated taking into consideration the STREGA recommendations (27).

\section{RESULTS}

The genotype frequency distributions were consistent with HWE ( $>0.3)$. All SNPs were grouped into a single haplotypic block (figure 1).

The characteristics of study subjects are shown in table 1 . In women there was no evidence for association between the genotypes and either BMI or BMD (table 2).

However, men with the minor $\mathrm{C}$ alleles at the rs 12049646 locus have a lower BMI ( $\mathrm{p}=0.0328$ under an additive model and 0.0296 under a dominant model). Likewise, those with $\mathrm{G}$ alleles at the neighbour locus rs3740051 tended to have a lower BMI, close to the statistical significance $(\mathrm{p}=0.0545$ under an additive model and 0.050 under a dominant model) (table 2). Similar results were observed in the haplotypic analysis. In multilocus models, only rs12049646 showed a significant independent effect. We found no association between the polymorphisms and BMD. Similar results were obtained in age-adjusted analyses (not shown).

Given the potential effects of sirtuins on bone cell differentiation, we also measured SIRTI messenger RNA in bone. SIRT1 expression was detected in all bone samples studied. The expression level was similar in samples from patients with fractures and in controls with osteoarthritis (3.4 \pm 3.9 relative units in fractures vs. $2.6 \pm 1.6$ in controls; $p=0.8$; figure 2 ). The association of rs12049646 and BMI was tested in a separate group of men, the populationbased Hortega cohort. In this group we found a similar trend for association between rs 12049646 alleles and BMI that was not statistically significant $(\mathrm{p}=0.266$; figure 3$)$. However, a relationship between the genotypes and BMI was confirmed in the combined analysis of both cohorts. In 
comparison with TT homozygotes, men with C alleles at the rs12049646 locus had lower BMI, with an averaged mean difference of $0.82 \mathrm{~kg} / \mathrm{m}^{2}$ (95\% confidence interval $\left.0.15-1.48 ; \mathrm{p}=0.016\right)$ (figure 3).

In order to confirm the potential regulatory role of the rs12049646 polymorphism, we studied the binding of nuclear proteins to both alleles by EMSA. As seen in figure 3, C alleles showed stronger binding properties than $\mathrm{T}$ alleles. In competition experiments with increasing amounts of unlabeled $\mathrm{C}$ allele oligonucleotide, the $\mathrm{T}$ allele oligonucleotide was more easily displaced from the complex when an excess of an unlabeled C oligonucleotide was used (figure 4). Taken together, the results indicated that the $\mathrm{C}$ to $\mathrm{T}$ change results in a reduced protein binding ability.

\section{DISCUSSION}

Body size, adiposity and bone mass have a strong genetic component. Incompletely elucidated interactions exist between these phenotypes. Several adipokines and hormones (including estrogens) synthesized in the adipose tissue may have effects on bone remodelling. It is also possible that common genetic factors influence both bone and body mass $(28 ; 29)$.

Sirtuins play an important role in energy metabolism regulation, and some experimental data also suggest an influence on bone remodelling. Therefore, we hypothesized that genetic variants of SIRT1 might be associated with differences in body and bone mass. The whole SIRT1 region is in strong linkage disequilibrium and in fact all the SNPs included in this study belong to a single haplotypic block. We could not find evidence for association between SIRT1 polymorphisms and BMD. Thus, our data argue against an important influence of allelic variants of the SIRT1 gene on bone mass. Additionally, no differences in SIRT1 expression were found between patients with fragility hip fractures and controls. Given the sample size in the discovery cohort, our study had $75 \%$ and $99 \%$ power (in men and women, respectively) to detect an allelic influence explaining at least $2 \%$ of BMD variation. However, the existence of allelic variants 
with smaller effects on BMD, or of rare variants with more important effects, cannot be excluded, particularly in men. For instance, the estimated power to detect a genetic effect explaining $0.5 \%$ of BMD variance was $26 \%$ in men and $46 \%$ in women.

On the other hand, a polymorphism located in the $5^{\prime}$ region of SIRT1 was associated with BMI in men. There was a consistent trend for the minor $\mathrm{C}$ alleles to be associated with lower BMI in the two cohorts studied, reaching the conventional threshold for statistical significance in one of them and in the combined analysis. The rs 12049646 polymorphism is located $20 \mathrm{~kb}$ upstream the translation start site. It could modulate SIRT1 transcription by influencing the binding of transcription factors or other regulatory molecules. In support of this notion, we found allelespecific differences in the binding of nuclear proteins in vitro. A bioinformatic analysis using MatInspector software (www.genomatix.de) revealed potential differences in the binding of some steroid hormone receptors. However, further experiments are needed to elucidate the transcription factors actually involved, as well as to confirm if an interaction between sex hormones and sirtuin 1 actually exists, which could explain the sex-related differences in the association between SIRT1 polymorphisms and BMI. Whatever the molecular explanation relating SNPs and gene transcription might be, differences in sirtuin expression may influence energy metabolism by several mechanisms, including the regulation of adipocyte differentiation and function $(3 ; 4 ; 30)$ and energy expenditure $(31 ; 32)$.

Genome-wide association studies (GWAS) have revolutionized the search of the genetic factors involved in obesity and other complex disorders. However, in a large collaborative analysis of GWAS results, SIRT1 was not pointed as a gene associated with BMI (17). Likewise, the GWAS catalog (www.genome.gov/gwastudies) does not include any study showing a significant association between SIRT1 polymorphisms and BMI. However, it does not exclude an influence of SIRT1 variants on BMI. Sample size, inclusion of heterogeneous groups of patients and other factors may limit the power of GWAS to identify some genes truly related to the phenotype 
studied. In fact, although GWAS have lead to the discovery of many genes not previously known to be associated with BMI, the proportion of the phenotypic variation explained by those genes is still very small. This indicates that much more research is needed to identify other gene variants and novel genes explaining the "missing heritability". Our results suggest that SIRT1 may be one of those genes, at least in male individuals.

Genetic association studies are prone to false-positive results derived from population stratification and other factors. The association between rs12049646 and BMI found in this study would not be statistically significant after multiple-test adjustment. However, we regarded it as likely real because it remained significant in the combined analysis of two independent cohorts and in vitro data were consistent with allelic differences in the binding of transcription factors. Data from other investigators also suggest an association between SIRT1 polymorphisms and adipose tissue mass. Zillikens reported an association of alleles of the noncoding rs 7895833 (also included in our study) and rs1467568 polymorphisms with BMI in the Dutch population (33). Van den Berg found an association of alleles at the synonymous SNP rs2273773 with BMI in other Dutch cohort (34). In a case-control study, Peeters genotyped two SNPs of the SIRTI gene and found an association of the intronic polymorphism rs7069102 with obesity (35). An association between several SIRT1 polymorphisms and BMI has also been recently reported in French and Swedish individuals (36). Likewise, in line with our results, a recent Japanese study found several polymorphisms associated with BMI in males, but not in females (37). On the other hand, an association between genetic variants of SIRT1 and energy expenditure was reported in a German study (38). Overall, these studies suggest an association of variants of the SIRT1 gene with adipose tissue mass and obesity. However, given the strong linkage in the region, it is hard to know which polymorphisms are actually responsible for the association. Deep-sequencing analyses of the whole region followed by further functional in vitro studies would be needed to elucidate the allelic variants actually responsible for the association. 
In conclusion, this study suggests that some common variants of the SIRT1 gene influence body mass index in a sex-specific way. However, we found no evidence for association with bone mineral density.

\section{ACKNOWLEDGEMENTS}

We acknowledge the excellent technical assistance of Carolina Sañudo, Verónica Mijares and Blanca Paule.

This study was supported by grants from Fundación Torres Quevedo-IDICAN-IFIMAV and Instituto de Salud Carlos III-FIS (PI08/0183).

\section{CONFLICTS OF INTEREST}

Authors do not have conflicts of interest relevant to this paper. 


\section{REFERENCES}

(1) $\mathrm{Yu}$ J, Auwerx J. The role of sirtuins in the control of metabolic homeostasis. Ann N Y Acad Sci 2009; 1173 Suppl 1:E10-E19.

(2) Rahman S, Islam R. Mammalian Sirt1: insights on its biological functions. Cell Commun Signal 2011; 9:11.

(3) Yamamoto H, Schoonjans K, Auwerx J. Sirtuin functions in health and disease. Mol Endocrinol 2007; 21:1745-1755.

(4) Pfluger PT, Herranz D, Velasco-Miguel S, Serrano M, Tschop MH. Sirt1 protects against high-fat diet-induced metabolic damage. Proc Natl Acad Sci U S A 2008; 105:97939798.

(5) Schug TT, Li X. Sirtuin 1 in lipid metabolism and obesity. Ann Med 2011; 43(3):198211.

(6) Schenk S, McCurdy CE, Philp A, Chen MZ, Holliday MJ, Bandyopadhyay GK et al. Sirt1 enhances skeletal muscle insulin sensitivity in mice during caloric restriction. J Clin Invest 2011; 121:4281-4288.

(7) Wang RH, Kim HS, Xiao C, Xu X, Gavrilova O, Deng CX. Hepatic Sirt1 deficiency in mice impairs mTorc2/Akt signaling and results in hyperglycemia, oxidative damage, and insulin resistance. J Clin Invest 2011; 121:4477-4490.

(8) Wei J, Ducy P. Co-dependence of bone and energy metabolisms. Arch Biochem Biophys 2010; 503:35-40.

(9) Clemens TL, Karsenty G. The osteoblast: an insulin target cell controlling glucose homeostasis. J Bone Miner Res 2011; 26:677-680.

(10) Backesjo CM, Li Y, Lindgren U, Haldosen LA. Activation of Sirt1 decreases adipocyte formation during osteoblast differentiation of mesenchymal stem cells. J Bone Miner Res 2006; 21:993-1002.

(11) Tseng PC, Hou SM, Chen RJ, Peng HW, Hsieh CF, Kuo ML et al. Resveratrol promotes osteogenesis of human mesenchymal stem cells by up-regulating RUNX2 gene expression via SIRT1/FOXO3A axis. J Bone Miner Res 2011; 26:2552-2563.

(12) Shakibaei M, Buhrmann C, Mobasheri A. Resveratrol-mediated SIRT-1 interactions with p300 modulate receptor activator of NF-kappaB ligand (RANKL) activation of NFkappaB signaling and inhibit osteoclastogenesis in bone-derived cells. J Biol Chem 2011; 286(13):11492-11505.

(13) Kupisiewicz K, Boissy P, Abdallah BM, Hansen FD, Erben RG, Savouret JF et al. Potential of resveratrol analogues as antagonists of osteoclasts and promoters of osteoblasts. Calcif Tissue Int 2010; 87:437-449. 
(14) Ralston SH. Osteoporosis as an hereditary disease. Clin Rev Bone Miner Metab 2010; 8:68-76.

(15) Loos RJ. Recent progress in the genetics of common obesity. Br J Clin Pharmacol 2009; 68:811-829.

(16) Richards JB, Kavvoura FK, Rivadeneira F, Styrkarsdottir U, Estrada K, Halldorsson BV et al. Collaborative meta-analysis: associations of 150 candidate genes with osteoporosis and osteoporotic fracture. Ann Intern Med 2009; 151:528-537.

(17) Speliotes EK, Willer CJ, Berndt SI, Monda KL, Thorleifsson G, Jackson AU et al. Association analyses of 249,796 individuals reveal 18 new loci associated with body mass index. Nat Genet 2010; 42(11):937-948.

(18) Olmos JM, Hernandez JL, Martinez J, Pariente E, Llorca J, Gonzalez-Macias J. Bone turnover markers in Spanish adult men The Camargo Cohort Study. Clinica Chimica Acta 2010; 411:1511-1515.

(19) Riancho JA, Olmos JM, Pineda B, Garcia-Ibarbia C, Perez-Nunez MI, Nan DN et al. Wnt receptors, bone mass, and fractures: gene-wide association analysis of LRP5 and LRP6 polymorphisms with replication. Eur J Endocrinol 2011; 164:123-131.

(20) Abellan R, Mansego ML, Martinez-Hervas S, Martin-Escudero JC, Carmena R, Real JT et al. Association of selected ABC gene family single nucleotide polymorphisms with postprandial lipoproteins: results from the population-based Hortega study. Atherosclerosis 2010; 211:203-209.

(21) Hernandez JL, Garcés CM, Sumillera M, Fernandez-Aldasoro EV, Garcia-Ibarbia C, Ortiz JA et al. Aromatase expression in osteoarthritic and osteoporotic bone. Arthritis Rheum 2008; 58:1696-1700.

(22) Velasco J, Zarrabeitia MT, Prieto JR, Perez-Castrillon JL, Perez-Aguilar MD, PerezNunez MI et al. Wnt pathway genes in osteoporosis and osteoarthritis: differential expression and genetic association study. Osteoporos Int 2010; 21:109-118.

(23) Ordovas L, Roy R, Pampin S, Zaragoza P, Osta R, Rodriguez-Rey JC et al. The g.763G $>$ C SNP of the bovine FASN gene affects its promoter activity via Sp-mediated regulation: implications for the bovine lactating mammary gland. Physiol Genomics 2008; 34:144-148.

(24) Heid IM, Jackson AU, Randall JC, Winkler TW, Qi L, Steinthorsdottir V et al. Metaanalysis identifies 13 new loci associated with waist-hip ratio and reveals sexual dimorphism in the genetic basis of fat distribution. Nat Genet 2010; 42(11):949-960.

(25) Barrett JC, Fry B, Maller J, Daly MJ. Haploview: analysis and visualization of LD and haplotype maps. Bioinformatics 2005; 21:263-265.

(26) Bax L, Yu LM, Ikeda N, Tsuruta H, Moons KG. Development and validation of MIX: comprehensive free software for meta-analysis of causal research data. BMC Medical Research Methodology 2006; 6:50. 
(27) Little J, Higgins JP, Ioannidis JP, Moher D, Gagnon F, von Elm E et al. STrengthening the REporting of Genetic Association studies (STREGA)--an extension of the STROBE statement. European Journal of Clinical Investigation 2009;247-266.

(28) Chen Y, Guo YF, Lei SF, Wang YB, Deng HW. Genetic and environmental correlations between bone mineral density and bone size in Caucasians. Hum Biol 2007; 79:15-24.

(29) Tang ZH, Xiao P, Lei SF, Deng FY, Zhao LJ, Deng HY et al. A bivariate whole-genome linkage scan suggests several shared genomic regions for obesity and osteoporosis. J Clin Endocrinol Metab 2007; 92:2751-2757.

(30) Fischer-Posovszky P, Kukulus V, Tews D, Unterkircher T, Debatin KM, Fulda S et al. Resveratrol regulates human adipocyte number and function in a Sirt1-dependent manner. Am J Clin Nutrit 2010; 92:5-15.

(31) Canto C, Auwerx J. PGC-1alpha, SIRT1 and AMPK, an energy sensing network that controls energy expenditure. Curr Opin Lipidol 2009; 20:98-105.

(32) Canto C, Gerhart-Hines Z, Feige JN, Lagouge M, Noriega L, Milne JC et al. AMPK regulates energy expenditure by modulating NAD+ metabolism and SIRT1 activity. Nature 2009; 458:1056-1060.

(33) Zillikens MC, van Meurs JB, Rivadeneira F, Amin N, Hofman A, Oostra BA et al. SIRT1 genetic variation is related to BMI and risk of obesity. Diabetes 2009; 58:28282834.

(34) van den Berg SW, Dolle ME, Imholz S, van der A DL, van 't Slot R, Wijmenga C et al. Genetic variations in regulatory pathways of fatty acid and glucose metabolism are associated with obesity phenotypes: a population-based cohort study. Int J Obes (Lond) 2009; 33:1143-1152.

(35) Peeters AV, Beckers S, Verrijken A, Mertens I, Roevens P, Peeters PJ et al. Association of SIRT1 gene variation with visceral obesity. Hum Genet 2008; 124:431-436.

(36) Clark SJ, Falchi M, Olsson B, Jacobson P, Cauchi S, Balkau B et al. Association of Sirtuin 1 (SIRT1) Gene SNPs and Transcript Expression Levels With Severe Obesity. Obesity (Silver Spring) 2012; 20:178-185.

(37) Shimoyama Y, Suzuki K, Hamajima N, Niwa T. Sirtuin 1 gene polymorphisms are associated with body fat and blood pressure in Japanese. Transl Res 2011; 157:339-347.

(38) Weyrich P, Machicao F, Reinhardt J, Machann J, Schick F, Tschritter O et al. SIRT1 genetic variants associate with the metabolic response of Caucasians to a controlled lifestyle intervention--the TULIP Study. BMC Med Genet 2008; 9:100. 
Table 1

Characteristics of study subjects

\begin{tabular}{llll}
\hline & $\begin{array}{l}\text { Cantabria, } \\
\text { males } \\
(\mathrm{n}=342)\end{array}$ & $\begin{array}{l}\text { Cantabria, } \\
\text { females } \\
(\mathrm{n}=1052)\end{array}$ & $\begin{array}{l}\text { Valladolid, } \\
\text { males } \\
(\mathrm{n}=408)\end{array}$ \\
\hline Age, yr & $67 \pm 7$ & $67 \pm 9$ & $70 \pm 11$ \\
Height, cm & $167 \pm 6$ & $155 \pm 6$ & $166 \pm 7$ \\
Weight, kg & $80 \pm 11$ & $68 \pm 12$ & $76 \pm 11$ \\
BMI, kg/m ${ }^{2}$ & $28.7 \pm 3.4$ & $28.3 \pm 4.6$ & $27.4 \pm 3.5$ \\
Type 2 diabetes, $\%$ & 14 & 18 & 16 \\
\hline
\end{tabular}

BMI, body mass index 
Table 2

Polymorphisms studied and association of genotypes and phenotypes in the discovery cohort.

\begin{tabular}{llllllll}
\hline & & & & \multicolumn{2}{c}{$\begin{array}{l}\text { p-values for } \\
\text { association in females }\end{array}$} & \multicolumn{2}{c}{$\begin{array}{l}\text { p-values for } \\
\text { association in males }\end{array}$} \\
\hline SNP & $\begin{array}{l}\text { Gene } \\
\text { location }\end{array}$ & $\begin{array}{l}\text { Minor } \\
\text { allele }\end{array}$ & MAF & BMD & BMI & BMD & BMI \\
\hline rs7895833 & 5 & G & 0.21 & 0.66 & 0.95 & 0.59 & 0.99 \\
rs17712705 & 5, & A & 0.32 & 0.76 & 0.70 & 0.42 & 0.39 \\
rs12049646 & 5, & C & 0.06 & 0.72 & 0.28 & 0.42 & $\underline{\mathbf{0 . 0 3}}$ \\
rs12778366 & 5, & C & 0.12 & 0.73 & 0.46 & 0.65 & 0.39 \\
rs3740051 & 5, & G & 0.06 & 0.79 & 0.23 & 0.49 & $\underline{\mathbf{0 . 0 5}}$ \\
rs10997860 & Intron & T & 0.33 & 0.91 & 0.53 & 0.27 & 0.57 \\
rs12413112 & Intron & A & 0.12 & 0.88 & 0.53 & 0.52 & 0.79 \\
rs10997866 & Intron & A & 0.35 & 0.69 & 0.62 & 0.29 & 0.78 \\
rs2224573 & Intron & A & 0.35 & 0.97 & 0.70 & 0.29 & 0.72 \\
rs10823111 & Intron & T & 0.33 & 0.86 & 0.50 & 0.29 & 0.53 \\
\hline
\end{tabular}

MAF: minor allele frequency

BMD: bone mineral density; BMI, body mass index

p-values under an additive model 


\section{FIGURE LEGENDS}

Figure 1. Linkage disequilibrium between SIRT1 polymorphisms. The numbers represent the D' distances (x100).

Figure 2. Sirtuin 1 gene expression in bone samples from patients with osteoporotic hip fractures and controls.

Figure 3. Differences in BMI between men with and without C alleles at the rs12049646 locus in the discovery and replication cohorts.

Figure 4. Differences in the binding of nuclear proteins to rs 12049646 alleles. A). Competitive EMSA's showing the differences in the protein binding ability as a consequence of the $\mathrm{C}$ to $\mathrm{T}$ change. Nuclear extracts were mixed with fluorescent oligonucleotides corresponding to either allele (the six lanes on the left correspond to the $\mathrm{C}$ allele and the six lanes on the right to the $\mathrm{T}$ allele) and competed with increasing amounts of unlabeled C- specific oligonucleotide. NE, no nuclear extract added to the reaction; the lanes labelled $\mathrm{C}$ and $\mathrm{T}$ indicated that no competitor was present; X10, X25 and X50 indicated an excess of 10, 25 or 50 fold of the unlabeled C- specific oligonucleotide. The specific bands are marked with arrows. B) Representation of the inverse of the upper band intensity versus unlabeled oligonucleotide excess. The slope of the line is inversely related to the protein binding ability of the oligonucleotide. 